\title{
IN SEARCH OF THE NEUROBIOLOGICAL BASIS OF DIVERGENT THINKING
}

\section{Alessandro Rossi, ${ }^{1}$ Giulia Sprugnoli, ${ }^{1}$ Emiliano Santarnecchi ${ }^{1-3}$}

\author{
${ }^{1}$ Brain Investigation \& Neuromodulation Laboratory, Department of Medicine, Surgery and Neuro- \\ science, University of Siena, Italy; ${ }^{2}$ Berenson-Allen Center for Non-Invasive Brain Stimulation, Beth Is- \\ rael Medical Center, Harvard Medical School, Boston, MA, USA; ${ }^{3}$ Center for Complex Systems Study, \\ Department of Engineering and Mathematics, University of Siena, Italy
}

\begin{abstract}
Human mind can follow two opposite type of reasoning in everyday life as well as in science: convergent thinking, as the ability to get the unique solution to a problem, or divergent thinking, the ability to elaborate different answer to a question. The latter is usually considered as an essential feature of the "creative mind", together with Insight, an unpredictable and unexpected moment of exceptional thinking commonly reported as the "Eureka!" experience. During such processing, an unconscious reorganization process of previously unrelated problem elements is made and when the solution finally emerges to consciousness, the subject is not able to explain how he/she reached it. Because of its unpredictable and unconscious nature, as well as its connection with creativity and scientific discoveries, the definition and evaluation of insight is now one of the biggest challenges for modern cognitive neuroscience. Neurophysiological evidence begins to arise, making the enhancement of creativity thinking using non-invasive neuromodulation techniques a plausible future scenario.
\end{abstract}

Key words: convergent thinking; divergent thinking; insight; neuromodulation.

\section{BACKGROUND}

Divergent thinking is commonly regarded as the "creative thinking", since Guilford defined it as the ability to find unusual and original answers to an open-ended problem, where no specific correct answer is provide [1]. This type of thinking is based on cognitive and executive functions like flexibility and inhibition (of consolidated ideas) that help finding new and creative responses to a posed question. Differently, convergent thinking is described as the ability to find the unique correct solution to a specific problem or question [2], requiring a discrete capacity of deductive reasoning and working memory to integrate the provided elements in a defined but not explicit pattern. Scientists mostly agree in considering divergent thinking and insight as the fundamental events that can lead to a creative idea.

Creativity is commonly considered the specific human ability to create something new, useful and generative [3] and it is regarded as the most important driving force of scientific, technological and artistic progress. While the identification of the neurobiological underpinning of fluid and crystallized intelligence is showing promising results thanks to straightforward testing tool for intelligence evaluation [4-7] creativity and insight still remain blurred concepts whose anatomical and functional definition still constitutes a challenge. Therefore, it is not surprising that research on creativity attracted many researchers but, giving the intrinsic difficulty on eliciting creative performance under controlled conditions, not much progress has been made so far [8].

Taken in account the little neurophysiological evidence about the underpinnings of divergent thinking, few experiments have been conducted with the noninvasive electrical stimulation in order to enhance individual performance on insight-related tasks. The possibility to modulate such an unexpected and unconscious generative event opens a new era of possibilities, not only restricted to neurophysiological research, but also of enormous importance to achieve a better understanding of the cognitive impairments accompanying several neurological and psychiatric disorders [9]. 


\section{INSIGHT: THEORY AND TASKS}

As everyone knows, there were numerous cases of insight in scientific discovery, for instance Newton's finding about the law of gravitation [10], but the very first insight moment is classically attributed to Archimedes of Syracuse, who ran naked down the street shouting "Eureka!" after the discovery of the buoyancy's principle while taking a bath [11].

This episode provides a simple and clear characterization of Aha moments, which was defined for the first time by the Gestalt psychologists as an unconscious and unpredictable reorganization of the problem components, that involved the discovery of a new mental representation [12]. The insight problem solving, as we can see, is almost opposite to the classical "search" or "analytic problem solving strategy", which is voluntary and completely conscious, with the subject being able to explain all the passages applied to reach the solution [13].

During the last century various definitions of insight were proposed by researchers, with the last iteration proposed by Kounios and Beeman [14] postulating intuition/insight as including "any sudden comprehension, realization, or problem solution that involves a reorganization of the elements of a person's mental representation of a stimulus, situation or event to yield a non obvious or non dominant interpretation". Moreover, in a framework supported by many other researchers [15] the Eureka moment is not strictly related to the field of problem solving but it includes also other domains of cognition, like perception and language comprehension.

Because of the various fields where the insight-like process appears to take place (identification of ambiguous visual stimuli, comprehension of metaphors, resolution of scientific problem), Bowden and colleagues also speculate that all these different types of Eureka moments share a basic neural network and the same mental steps, whereas specific subcomponents allow to separate and identify the various "type" of insight and also to differentiate them from the analytical method [15].

As, intuition moment is considered a subcomponent of the creativity process, together with the divergent thinking and the artistic creativity [8]. However, the demarcation between creativity and these mental creativity elements are not so clear, despite many studies focused on them. Insight is often seen as the first step of the creative idea generation, since it happened in a lots of scientific discoveries, like the Newton episode with the apple fall from the tree and even the elaboration of relativity theory by Einstein. With these premises, we can understand the similarity of insight and creativity task using in literature to assess these two related pattern of thinking. RAT (Remote Associates Test) and the CRA (Compound remote associate task) tasks represent a classical example of such overlap. Created in the Sixties to evaluate the ability of identifying semantic distant association between pro- vided words, RAT was used to assess the creativity and recently also for insight performance [16]. CRA were developed by Bowden in order to refine the RAT task, but with the aim of assessing the insight problem solving and not creativity in general. Indeed, CRA and RAT need to be solved with both divergent and convergent thinking: the former is aimed at exploring the words semantically related to the others provided, while the convergent component is leveraged to choose the unique solution. However, the most used task to assess creativity (Alternate Uses Task, AUT) is a pure divergent thinking task [17]. In our opinion, literature needs separate and different task to evaluate insight and creativity, because they are clearly not the same cognitive process, even if they seem to be really interconnected.

Finally, despite the fact that the insight is an unconscious and unpredictable process, Bowden and Beeman tried to propose a coherent sequence of events that could lead to intuition. First, they identify i) a strong conscious activation of information consolidated in the mental network of the subject accompanied by a weak and unconscious activation of information not directly related to the solutions in the initial subject's mental representation of the problem. Second, the activation of weak and secondary information conduct the subject to ii) a restructuration of the elements that allow the person to reach a new vision of the problem; finally, the appearance of the solution to consciousness elicits iii) a sense of enlightenment that was manifested with the typical "Aha!" exclamation [18].

The network that could support this process may be grounded in the hemispheric asymmetry: the initial weak sematic activation could take place in the right hemisphere, a process traditionally related to a general sematic coding process; on the contrary, it seems more likely that the dominant interpretation occurs the left hemisphere, which is involved in subtle sematic coding processes $[15,18]$. Given the recent evidence that relates the activation of right anterior superior temporal gyrus evaluate distant sematic relations between provided words [19] the restructuration process is considered to happen in this very cortical region.

As we can imagine, literature is just proposing the first evidence that could validate the last hypothesis on the neural substrates and functional steps for intuition. First of all, many researchers postulate that the main hemisphere where the insight events take place is the right because of its coarse semantic coding [15]. Moreover, Beeman and colleagues demonstrate specific activations of cortical regions during the resolution of CRA that support this process localization, specifically they revealed a primary activation in the right parietal lobe while the subject try to solve the task (and probably operate the reorganization of the problem's elements), followed by a shift of activation in the right temporal cortex when the solution emerges to the consciousness. 
Giving thanks to recent development in electrophysiological and imaging techniques, identification the neural basis and the time series of insight events seems to be an imminent achievable goal.

\section{MODULATION OF INSIGHT ABILITIES}

Neuromodulation techniques are relatively recent non-invasive methods to interact with cognitive functions both in the healthy and pathological brain. They include transcranial electrical stimulation (tES) and transcranial magnetic stimulation (TMS). In the last 1985 TMS technique was used to evaluate the relevance of specific brain region in cognitive functions, pathophysiology of various neurologic and psychiatric disorders, leading to a consistent increase of clinical application field [20]. Transcranial electrical stimulation, developed more recently, was currently employed to explore the neural correlates of intelligence, executive functions and motor performance with first evidences of its utility in cognitive impairments [4].

tES allows using different type of electrical current delivered via electrode placed on the scalp, such as direct current in the case of tDCS (transcranial direct current stimulation, inducing either an excitatory or inhibitory effect), alternating current in tACS (transcranial alternating current stimulation), or random-noise in tRNS (transcranial random noise current stimulation). Despite the specific mechanism of every electrical stimulations, in all of them we couldn't determine a real fire of neurons under the electrode, but increment the probability that it could happen. In other words, we try to facilitate the activation of a selected cortex important for specific mental process, entering in communication with the brain region via its specific "language" (the electrical current). The TMS method is similar to tES, since it induces an electrical field on the cortex derived from the magnetic stimulation. The difference is that with this technique we can effectively induce a neuronal fire and that the stimulation site is more focal in the brain. TMS is now used to treat many neuropsychiatric illness such depression, bipolar disorder and Parkinson disease and the possibility of clinical applications is still increasing. Regarding the transcranial electrical stimulation studies, there are only three papers that used tDCS over the prefrontal cortex and two studies that stimulate the temporal cortex.

As for the temporal lobe, electrical stimulation (tDCS) was applied to the right anterior temporal lobe and lead to an increase in number of participants solving the task, in contrast to placebo stimulation (so called "sham") and stimulation of the left anterior temporal lobe. These findings are in agreement with the hypothesis that insight-related processing primarily involves the right hemisphere. However, there are limits for the reliability of this data because of the insighttask used, which was a single problem in the second study [21], and a very small set of problems of the same type in the first study [22]. Moving away from the temporal lobe, the first study applying tDCS over the prefrontal cortex was conducted by Cerruti [16], testing the effect of excitatory stimulation on the right and left dorsolateral prefrontal cortex. Results shown an improvement on the RAT score only for the stimulation conducted over left dorsolateral prefrontal cortex.

Afterwards, in Metuki et al. 2012 [23], excitatory stimulation of the left prefrontal cortex was shown to enhance the performance during the solution of CRA problems, selectively for the most difficult trials. Finally, Chrysikou et al. 2013 [24] tested inhibitory stimulation (i.e. catodal tDCS) over the right and left prefrontal cortex, during a uncommon uses generation task. Results demonstrated that only inhibitory stimulation on left prefrontal cortex enhance performance.

As it is clearly visible, there are just a handful of studies assessing the possibility to modulate insight abilities in humans, mostly focusing on the prefrontal and temporal lobe by only using direct current stimulation protocols. Most importantly, results seem concordant only for stimulation over the temporal lobe, while positive effects are present both for excitatory and inhibitory stimulation. Overall, the left prefrontal cortex seems to be more implicated in the insight process than the right prefrontal cortex.

For what concerned TMS, only one study about the perception of degraded images is available to date. Results shown that right and left prefrontal TMS reduced the performance of healthy subjects, with results being in contrast with previously cited experiments using tDCS on the prefrontal cortex, even though the task were different.

\section{CONCLUSIONS}

Research on insight is really fascinating thanks to its characteristic unpredictability and unconscious nature. Despite the scientific interest on this mental process is constantly increasing we are still facing strong challenges in its definition, measurement and potentially, enhancement. Latest development in imaging techniques and electrophysiological investigations makes feasible to enlight the neural mechanism of insight, with benefits spanning from the study of developmental disorders to ageing processes. Moreover, the promising results of recent neuromodulation protocols open new questions about the possible use of electrical and magnetic stimulation to enhance problems solving ability in pathological conditions and to boost the physiological cognitive limits of healthy subjects, with strong ethical issues to be considered. 


\section{REFERENCES}

1. Guilford JP. The nature of human intelligence. New York: McGraw-Hill; 1967.

2. Abraham A, Windmann S. Creative cognition: the diverse operations and the prospect of applying a cognitive neuroscience perspective. Methods 2007;42:38-48.

3. Sternberg RJ, Davidson JE. The nature of insight. Cambridge: MIT Press; 1995.

4. Santarnecchi E, Polizzotto NR, Godone M, et al. Frequency-dependent enhancement of fluid intelligence induced by transcranial oscillatory potentials. Curr Biol 2013;23:1449-53.

5. Santarnecchi E, Galli G, Polizzotto NR, et al. Efficiency of weak brain connections support general cognitive functioning. Human Brain Mapp 2014;35:4566-82.

6. Santarnecchi E, Tatti E, Rossi S, et al. Intelligence-related differences in the asymmetry of spontaneous cerebral activity. Human Brain Mapp 2015;36:3586-602.

7. Santarnecchi E, Rossi S, Rossi A. The smarter, the stronger: intelligence level correlates with brain resilience to systematic insults. Cortex 2015;64:293-309.

8. Dietrich A, Kanso R. A review of EEG, ERP, and neuroimaging studies of creativity and insight. Psychol Bull 2010;136:822-48.

9. Santarnecchi E, Brem AK, Levenbaum E, et al. Enhancing cognition using transcranial electrical stimulation. Curr Opin Behav Sci 2015:4:171-8.

10. Sandkühler S, Bhattacharya J. Deconstructing insight: EEG correlates of insightful problem solving. PLoS One 2008;3:e1459.

11. Aziz-Zadeh L, Kaplan JT, Iacoboni M. Aha!: the neural correlates of verbal insight solutions. Human Brain Mapp 2009;30:908-16.

12. Kohler W. The mentality of apes. London: Routledge $\theta$ Kegan Paul; 1925.

13. Novick LR, Sherman SJ. On the nature of insight solutions: evi- dence from skill differences in anagram solution. Q J Exp Psychol A 2003;56:351-82.

14. Beeman M, Kounios J. The cognitive neuroscience of insight. Ann Rev Psychol 2014;65:71-93.

15. Bowden EM, Jung-Beeman M. Methods for investigating the neural components of insight. Methods 2007;42:87-99.

16. Cerruti C, Schlaug G. Anodal transcranial direct current stimulation of the prefrontal cortex enhances complex verbal associative thought. J Cogn Neurosci 2009;21:1980-7.

17. Wallach MA, Koogan N. Modes of thinking in young children: a study of the creativity. Intelligence distinction. New York: Holt, Rinehart $\theta$ Winston; 1965.

18. Bowden EM, Jung-Beeman M, Fleck J, Kounios J. New approaches to demystifying insight. Trends Cogn Sci 2005;9:322-8.

19. Mashal N, Faust M, Hendler T. The role of the right hemisphere in processing nonsalient metaphorical meanings: application of principal components analysis to fMRI data. Neuropsychologia 2005:43:2084-100.

20. Rossi S, Hallett M, Rossini PM, Pascual-Leone A. Safety of TMS consensus group. Safety, ethical considerations, and application guidelines for the use of transcranial magnetic stimulation in clinical practice and research. Clin Neurophysiol 2009;120:2008-39.

21. Chi RP, Snyder AW. Brain stimulation enables the solution of an inherently difficult problem. Neurosci Lett 2012;515:121-4.

22. Chi RP, Snyder AW. Facilitate Insight by non-invasive brain stimulation. PLoS One 2011;6:e16655.

23. Metuki N, Sela T, Lavidor M. Enhancing cognitive control components of insight problems solving by anodal tDCS of the left dorsolateral prefrontal cortex. Brain Stimul 2012;5:110-5.

24. Chrysikou EG, Hamilton RH, Coslett HB, et al. Noninvasive transcranial direct current stimulation over the left prefrontal cortex facilitates cognitive flexibility in tool use. Cogn Neuroscience 2013; 4:81-9. 